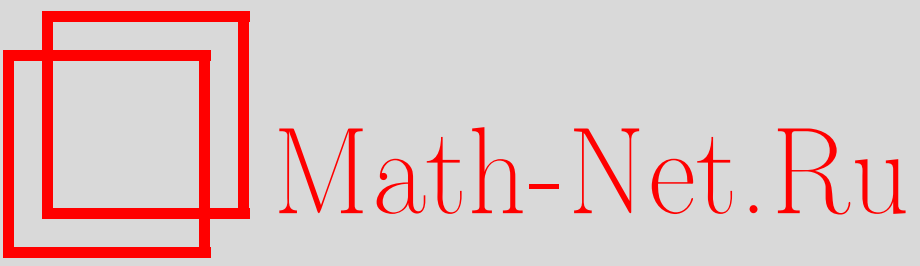

В. П. Маслов, А. Э. Рууге, Многочастичный и квазиклассический предельные переходы для нерелятивистских бозонов в квантованном электромагнитном поле, ТМФ, 2000, том 125, номер 3, 453-470

DOI: https://doi.org/10.4213/tmf679

Использование Общероссийского математического портала Math-Net.Ru подразумевает, что вы прочитали и согласны с пользовательским соглашением

http: //www.mathnet.ru/rus/agreement

Параметры загрузки:

IP : 54.166 .219 .16

26 апреля 2023 г., 14:55:14 


\section{МНОГОЧАСТИЧНЫЙ И КВАЗИКЛАССИЧЕСКИЙ ПРЕДЕЛЬНЫЕ ПЕРЕХОДЫ ДЛЯ НЕРЕЛЯТИВИСТСКИХ БОЗОНОВ В КВАНТОВАННОМ ЭЛЕКТРОМАГНИТНОМ ПОЛЕ}

\footnotetext{
С помощью метода комплексного ростка получена система уравнений для амплитуд одночастичных фазовых плотностей системы, состоящей из нескольких сортов классических частиц с электромагнитным взаимодействием. Соответствующие уравнения возникают в результате весьма сложного предельного перехода не из классических уравнений для $N$ частиц в магнитном поле, а из теории взаимодействия бозонов с квантованным электромагнитным полем, из двойной асимптотики: во-первых, по большому числу частиц и форотонов, а во-вторых, в квазиклассическом пределе. При этом в вышеуказанном первом пределе при некоторых дополнительных предположениях получаются уравнения, являющиеся условием стационарности функционала действия, рассматривавшегося в одной из недавних работ Фаддеевым и Ниеми.
}

\section{1. ВВЕДЕНИЕ}

В работе [1] были построены асимптотические решения вторично квантованного уравнения Лиувилля для системы большого числа классических тождественных попарно взаимодействуюших между собой частиц, находяшихся в заданном внешнем поле $U(x)$, $x \in \mathbb{R}^{3}$, отвечающие предельному переходу по параметру взаимодействия $\varepsilon \rightarrow 0$. Это уравнение имеет вид

$$
\begin{gathered}
i \frac{\partial \Phi(t)}{\partial t}=\widehat{\mathcal{H}} \Phi(t), \quad \Phi(t) \in \Gamma_{s}\left(L^{2}\left(\mathbb{R}^{3} \times \mathbb{R}^{3}\right)\right), \\
\widehat{\mathcal{H}}=-i \iint d x d p u^{+}(x, p)\left\{\frac{p^{2}}{2 m}+U(x), u^{-}(x, p)\right\}- \\
-\frac{i \varepsilon}{2} \iiint \int d x d p d \xi d \eta u^{+}(x, p) u^{+}(\xi, \eta)\left\{V(x-\xi), u^{-}(\xi, \eta) u^{-}(x, p)\right\},
\end{gathered}
$$

где $\Gamma_{s}\left(L^{2}\left(\mathbb{R}^{3} \times \mathbb{R}^{3}\right)\right)$ - симметричное пространство Фока [2] с одночастичным подпространством $L^{2}\left(\mathbb{R}^{3} \times \mathbb{R}^{3}\right), u^{ \pm}(x, p)$ - операторы рождения-уничтожения в этом пространстве, $x, p \in \mathbb{R}^{3}, t \in \mathbb{R},\{\cdot, \cdot\}$ - скобки Пуассона, $m$ - масса частиц, $\varepsilon V(x-\xi)$ - потенциал

\footnotetext{
* Московский государственный университет, Москва, Россия
} 
взаимодействия между частицами, функции $U$ и $V$ предполагаются достаточно гладкими.

Основная идея, с помощью которой строились асимптотические решения уравнения (1), состояла в следующем. Малый параметр $\varepsilon$ входит в оператор $\widehat{\mathcal{H}}(2)$ таким образом, что его можно записать в виде

$$
\widehat{\mathcal{H}}=\frac{1}{\varepsilon} \mathcal{H}\left(\sqrt{\varepsilon} u^{+}, \sqrt{\varepsilon} u^{-}\right)
$$

(операторы рождения $u^{+}$действуют после операторов уничтожения $u^{-}$). Отсюда видно, что если перейти к шредингеровскому представлению [2],

$$
u^{ \pm}(x, p) \rightarrow \frac{1}{\sqrt{2 \varepsilon}}\left(Q(x, p) \mp \varepsilon \frac{\delta}{\delta Q(x, p)}\right),
$$

то уравнение (1) примет вид уравнения Шредингера с бесконечным числом степеней свободы, в котором при операторах дифференцирования стоит мальй параметр $\varepsilon$. K этому уравнению применим один из методов построения квазиклассических асимптотик - метод комплексного ростка [3], используемый в квантовой механике конечного числа переменных для построения квазиклассических асимптотических решений уравнения Шредингера, сосредоточенных в малой $(\sim \sqrt{\hbar}, \hbar \rightarrow 0)$ окрестности подмногообразий конфигурационного пространства. Роль параметра квазиклассического разложения в данном случае играет параметр взаимодействия $\varepsilon$, а роль конфигурационного пространства играет множество действительных элементов пространства $L^{2}\left(\mathbb{R}^{3} \times \mathbb{R}^{3}\right)$.

Простейший вариант теории комплексного ростка - комплексньй росток в точке позволяет построить асимптотические решения уравнения Шредингера, сосредоточенные в каждый момент времени $t$ вблизи точки. Траектория движения этой точки представляет собой проекцию на конфигурационное подпространство одной из фазовых траекторий соответствуюшей классической гамильтоновой системы. Применительно к рассматриваемому случаю систему Гамильтона удобно записать в виде одного уравнения

$$
\frac{\partial}{\partial t} u(x, p)=-\left\{\frac{p^{2}}{2 m}+U(x), u(x, p)\right\}-\iint d \xi d \eta \bar{u}(\xi, \eta)\{V(x-\xi), u(\xi, \eta) u(x, p)\},
$$

где $u(x, p)=(Q(x, p)+i P(x, p)) / \sqrt{2} \in L^{2}\left(\mathbb{R}_{x}^{3} \times \mathbb{R}_{p}^{3}\right), Q(x, p)$ и $P(x, p)$ - классические координаты и канонически сопряженные им импульсы, соответствуюшие оператору умножения на $Q(x, p)$ и оператору дифференцирования $-i \varepsilon \delta / \delta Q(x, p)$ (в обозначении $u, Q$ и $P$ для краткости опускается аргумент $t$ ).

Гамильтонова система (3) позволяет получить хорошо известное уравнение Власова

$$
\begin{gathered}
\frac{\partial}{\partial t} \rho(x, p, t)=-\left\{H_{\rho}(x, p, t), \rho(x, p, t)\right\}, \\
H_{\rho}(x, p, t)=\frac{p^{2}}{2 m}+U(x)+\iint d \xi d \eta V(x-\xi) \rho(\xi, \eta, t) .
\end{gathered}
$$


Именно, каждому решению $u$ уравнения (3) соответствует решение $\rho=|u|^{2}$ уравнения (4). Таким образом, решение $u(x, p)$ уравнения (3) является амплитудой одночастичной плотности $\rho$.

В настояшей работе ставится задача обобшить уравнение (3) для системы большого числа частищ различных типов с электромагнитным взаимодействием. На первый взгляд может показаться, что данное обобщение очевидно. Так, если имеется всего один сорт частиц, то, казалось бы, нужно просто заменить импульсы $p$ на $p-e A(x, t) / c$, где $e$ - заряд частишы, $c$ - скорость света, $A(x, t)$ - вектор-потенциал магнитного поля, а в качестве потенциала $V(x-\xi)$ (предполагая, что задача рассматривается в кулоновской калибровке [4]) подставить $e^{2} /|x-\xi|$. Интеграл в правой части (5), деленный на заряд $e$, имел бы в этом случае смысл самосогласованного электрического потенциала $\Phi(x, t)$, удовлетворяюшего уравнению

$$
\Delta \Phi(x, t)=-4 \pi e \int d p \rho(x, p, t)
$$

По аналогии с этим можно было бы заключить, что вектор-потенциал магнитного поля $A(x, t)$ должен удовлетворять уравнению

$$
\left(\frac{1}{c^{2}} \frac{\partial^{2}}{\partial t^{2}}-\Delta\right) A(x, t)-\frac{1}{c} \nabla \frac{\partial}{\partial t} \Phi(x, t)=\frac{4 \pi e}{c} j(x, t),
$$

в котором в правой части в качестве вектора тока $j(x, t)$ следует взять вектор с компонентами

$$
j_{l}(x, t)=\frac{1}{m} \int d p\left(p_{l}-\frac{e}{c} A_{l}(x, t)\right) \rho(x, p, t), \quad l=1,2,3 .
$$

Тем не менее подобное рассуждение дает правильное обобщение уравнения для $\rho$ (уравнение Власова с магнитным полем)

$$
\frac{\partial}{\partial t} \rho(x, p, t)=-\left\{\frac{1}{2 m} \sum_{j=1}^{3}\left(p_{j}-\frac{e}{c} A_{j}(x, t)\right)^{2}+e \Phi(x, t), \rho(x, p, t)\right\},
$$

но не уравнения для $u$. Дело в том, что если положить $\rho=|u|^{2}$ и (формально) выразить из уравнений (6) и (7) потенциалы электромагнитного поля в терминах $\rho$, а затем подставить их в модифицированное указанным образом уравнение для $u$ (3), то получаюшаяся система уравнений для $u$ уже не будет гамильтоновой, т.к. в нее будут входить значения $u$, взятые в различные моменты времени $t$, между тем как гамильтоновость необходима по смыслу. Это означает, что для построения обобшения уравнения (3) необходимо учитывать переменные, соответствуюшие электромагнитному полю, наравне с переменными $\operatorname{Re} u$ и $\operatorname{Im} u$. Этому более тонкому анализу и посвящена настоящая работа. 
Окончательные уравнения имеют вид

$$
\begin{aligned}
& \frac{\partial}{\partial t} u_{a}(x, p)=-\left\{\frac{1}{2 m_{a}} \sum_{j=1}^{3}\left(p_{j}-\frac{e_{a}}{c} A_{j}(x)\right)^{2}+e_{a} \Phi(x), u_{a}(x, p)\right\}+ \\
& +i\left[\frac{e_{a}}{m_{a} c} \sum_{j=1}^{3}\left(p_{j}-\frac{e_{a}}{c} A_{j}(x)\right) \tilde{A}_{j}(x)+e_{a} \widetilde{\Phi}(x)\right] u_{a}(x, p), \\
& \Delta \Phi(x)=-4 \pi \sum_{a=1}^{a_{0}} e_{a} \int d p\left|u_{a}(x, p)\right|^{2}, \\
& \left(\frac{1}{c^{2}} \frac{\partial^{2}}{\partial t^{2}}-\Delta\right) A_{j}(x)-\frac{1}{c} \frac{\partial^{2}}{\partial x_{j} \partial t} \Phi(x)= \\
& =\frac{4 \pi}{c} \sum_{a=1}^{a_{0}} \frac{e_{a}}{m_{a}} \int d p\left(p_{j}-\frac{e_{a}}{c} A_{j}(x)\right)\left|u_{a}(x, p)\right|^{2}, \\
& \Delta \widetilde{\Phi}(x)=i 4 \pi \sum_{a=1}^{a_{0}} e_{a} \int d p\left\{u_{a}(x, p), \bar{u}_{a}(x, p)\right\}, \\
& \left(\frac{1}{c^{2}} \frac{\partial^{2}}{\partial t^{2}}-\Delta\right) \tilde{A}_{j}(x)-\frac{1}{c} \frac{\partial^{2}}{\partial x_{j} \partial t} \widetilde{\Phi}(x)= \\
& =-\frac{4 \pi}{c} \sum_{a=1}^{a_{0}} \frac{e_{a}}{m_{a}}\left(i \int d p\left(p_{j}-\frac{e_{a}}{c} A_{j}(x)\right)\left\{u_{a}(x, p), \bar{u}_{a}(x, p)\right\}+\right. \\
& \left.+\frac{e_{a}}{c} \tilde{A}_{j}(x) \int d p\left|u_{a}(x, p)\right|^{2}\right), \\
& \sum_{j=1}^{3} \frac{\partial}{\partial x_{j}} A_{j}(x)=0, \quad \sum_{j=1}^{3} \frac{\partial}{\partial x_{j}} \tilde{A}_{j}(x)=0,
\end{aligned}
$$

где в обозначениях $u_{a}$ и функций $A_{j}, \Phi, \tilde{A}_{j}$ и $\widetilde{\Phi}$ опушен для краткости аргумент $t, a=$ $\overline{1, a_{0}}$ - индекс, нумерующий различные типы частиц, $u_{a} \in L^{2}\left(\mathbb{R}_{x}^{3} \times \mathbb{R}_{p}^{3}\right), e_{a}$ и $m_{a}$ - соответственно заряд и масса частицы типа $a$. Несложно видеть, что $\rho_{a}=\left|u_{a}\right|^{2}$ будут удовлетворять системе уравнений Власова с магнитным полем

$$
\frac{\partial}{\partial t} \rho_{a}(x, p)=-\left\{\frac{1}{2 m_{a}} \sum_{j=1}^{3}\left(p_{j}-\frac{e_{a}}{c} A_{j}(x)\right)^{2}+e_{a} \Phi(x), \rho_{a}(x, p)\right\} .
$$

Отметим, что если $u_{a}(x, p)$ - не обрашаюшаяся в ноль функция, то можно сделать замену $u_{a}=\sqrt{\rho_{a}} e^{i \varphi_{a}}$. Уравнение для фазы $\varphi_{a}(x, p)$ будет иметь вид

$$
\begin{aligned}
\frac{\partial}{\partial t} \varphi_{a}(x, p)= & -\left\{\frac{1}{2 m_{a}} \sum_{j=1}^{3}\left(p_{j}-\frac{e_{a}}{c} A_{j}(x)\right)^{2}+e_{a} \Phi(x), \varphi_{a}(x, p)\right\}+ \\
& +\frac{e_{a}}{m_{a} c} \sum_{j=1}^{3}\left(p_{j}-\frac{e_{a}}{c} A_{j}(x)\right) \tilde{A}_{j}(x)+e_{a} \widetilde{\Phi}(x),
\end{aligned}
$$


где, как и выше, опущен аргумент $t$ в обозначениях функций.

Ниже будет показано, что уравнения (9)-(14) эквиваленты системе Гамильтона, которая возникает из уравнений теории взаимодействия частищ с квантованным электромагнитным полем в результате двух “классических" предельных переходов: сначала делается предельный переход, при котором среднее число частиц и фотонов в системе стремится к бесконечности (в качестве параметра квазиклассического разложения при этом выступает величина, обратная среднему общему числу частиц в системе), а затем $\hbar \rightarrow 0$.

\section{2. НЕКОТОРЫЕ ФОРМУЛЫ МЕТОДА ВТОРИЧНОГО КВАНТОВАНИЯ}

Напомним, что под симметричным пространством Фока $\Gamma_{s}\left(L^{2}(\mathcal{M})\right)$, где $\mathcal{M}$ - некоторое пространство с мерой, понимается гильбертово пространство, элементами которого являются бесконечные последовательности

$$
\begin{gathered}
\Phi=\left(\Phi_{0}, \Phi_{1}\left(z_{1}\right), \Phi_{2}\left(z_{1}, z_{2}\right), \ldots\right), \\
\Phi_{0} \in \mathbb{C}, \quad \Phi_{n}\left(z_{1}, \ldots, z_{n}\right) \in L^{2}(\underbrace{\mathcal{M} \times \cdots \times \mathcal{M}}_{n \text { раз }}),
\end{gathered}
$$

такие, что $\Phi_{n}$ симметричны относительно перестановок $z_{i} \rightleftarrows z_{j}$ и выполнено условие

$$
\|\Phi\|^{2}=\left|\Phi_{0}\right|^{2}+\sum_{n=1}^{\infty} \int d z_{1} \ldots d z_{n}\left|\Phi_{n}\left(z_{1}, \ldots, z_{n}\right)\right|^{2}<\infty .
$$

Определение операторов рождения-уничтожения $\psi^{ \pm}(z)$, действуюших в этом пространстве, имеет вид

$$
\begin{gathered}
\left(\psi^{+}(z) \Phi\right)_{n}\left(z_{1}, \ldots, z_{n}\right)=\frac{1}{\sqrt{n}} \sum_{i=1}^{n} \delta\left(z-z_{i}\right) \Phi_{n-1}\left(z_{1}, \ldots, z_{i-1}, z_{i+1}, \ldots, z_{n}\right), \\
\left(\psi^{-}(z) \Phi\right)_{n-1}\left(z_{1}, \ldots, z_{n-1}\right)=\sqrt{n} \Phi_{n}\left(z_{1}, \ldots, z_{n-1}, z\right) .
\end{gathered}
$$

Операторы рождения-уничтожения удовлетворяют коммутационным соотношениям

$$
\left[\psi^{-}(z), \psi^{+}(\zeta)\right]=\delta(z-\zeta), \quad\left[\psi^{ \pm}(z), \psi^{ \pm}(\zeta)\right]=0
$$

Элемент $\Phi_{n}$ последовательности (16) называется $n$-частичной компонентой фоковского вектора $\Phi$, а подпространство в $\Gamma_{s}\left(L^{2}(\mathcal{M})\right)$, образованное элементами, у которых лишш $n$-частичная компонента отлична от нуля, называется $n$-частичныл странством. Элемент $\Phi_{\mathrm{vac}}=(1,0,0, \ldots)$ называется вакуумным вектором. Имеет место следующая формула, представляющая фоковский вектор (16) через операторы рождения:

$$
\Phi=\sum_{N=1}^{\infty} \frac{1}{\sqrt{N !}} \int d z_{1} \ldots d z_{N} \Phi_{N}\left(z_{1}, \ldots, z_{N}\right) \psi^{+}\left(z_{1}\right) \ldots \psi^{+}\left(z_{N}\right) \Phi_{\mathrm{vac}}
$$


В шредингеровском представлении [2] элементам пространства Фока соответствуют функционалы $\Phi[Q(\cdot)], Q=Q^{*} \in L^{2}(\mathcal{M})$, причем вакуумному вектору ставится в соответствие функционал

$$
\Phi_{\mathrm{vac}}[Q(\cdot)]=\exp \left\{-\frac{1}{2 \varepsilon} \int_{\mathcal{M}} d z Q^{2}(z)\right\},
$$

где $\varepsilon>0$ - параметр. Операторам рождения-уничтожения соответствуют операторы

$$
\psi_{Q}^{ \pm}(z)=\frac{1}{\sqrt{2 \varepsilon}}\left(Q(z) \mp \varepsilon \frac{\delta}{\delta Q(z)}\right) .
$$

Функционал, соответствующий вектору обшего вида (16), можно вычислить с помощью формулы $(17)$, заменяя в ее правой части $\Phi_{\mathrm{vac}}$ на $\Phi_{\mathrm{vac}}[Q(\cdot)]$ и $\psi^{+}(z)$ на $\psi_{Q}^{+}(z)$.

\section{3. ВЫВОД УРАВНЕНИЯ ДЛЯ АМПЛИТУДЫ ИЗ КВАНТОВЫХ УРАВНЕНИЙ}

Как уже говорилось во введении, для того чтобы построить обобшение уравнения (3), необходимо включить в рассмотрение переменные, соответствующие электромагнитному полю, наравне с переменными $\operatorname{Re} u$ и $\operatorname{Im} u$, для чего, в свою очередь, необходимо перейти к квантовым уравнениям.

Уравнение (3) было получено из классического $(\hbar=0)$ вторично квантованного уравнения (1) в результате предельного перехода по параметру взаимодействия $\varepsilon \rightarrow 0$. Покажем теперь, каким образом его можно получить исходя из квантовых уравнений, осуществляя предельный переход сначала по параметру взаимодействия между частицами $\varepsilon \rightarrow 0$, а затем по $\hbar \rightarrow 0$.

Будем рассматривать систему, состояшую из тождественных бесспиновых бозонов. Вторично квантованное уравнение Шредингера, описывающее эту систему, имеет вид

$$
i \frac{\partial}{\partial t} \Psi(t)=\frac{1}{\varepsilon} H\left(\sqrt{\varepsilon} \psi^{2}+\sqrt{\varepsilon} \psi^{-}\right) \Psi(t), \quad \Psi(t) \in \Gamma_{s}\left(L^{2}\left(\mathbb{R}^{3}\right)\right),
$$

где индексы над $\psi^{+}$и $\psi^{-}$обозначают порядок действия операторов рождения и уничтожения [5],

$$
\begin{aligned}
H\left(\psi^{*}, \psi\right)= & \frac{1}{\hbar} \int d x \psi^{*}(x)\left(-\frac{\hbar^{2}}{2 m} \Delta+U(x)\right) \psi(x)+ \\
& +\frac{1}{2 \hbar} \iint d x d y V(x-\xi) \psi^{*}(x) \psi^{*}(y) \psi(y) \psi(x)
\end{aligned}
$$

$U(x)$ - заданный внешний потенциал, $V(x-y)$ - потенциал взаимодействия между частицами, деленный на параметр взаимодействия $\varepsilon$; функции $U$ и $V$ для простоты предполагаются достаточно гладкими. Гамильтонову систему, соответствуюшую пределу $\varepsilon=0$, можно записать в виде

$$
\begin{aligned}
i \frac{\partial}{\partial t} \psi(x) & =\frac{\delta H\left(\psi^{*}, \psi\right)}{\delta \psi^{*}(x)}= \\
& =\frac{1}{\hbar}\left(-\frac{\hbar^{2}}{2 m} \Delta+U(x)\right) \psi(x)+\frac{1}{\hbar} \int d y V(x-y) \psi^{*}(y) \psi(y) \psi(x)
\end{aligned}
$$


где для краткости у $\psi$ опушен аргумент $t, \psi \in L^{2}\left(\mathbb{R}_{x}^{3}\right)$.

Рассмотрим теперь два каких-нибудь решения, например $\psi(x, t)$ и $\phi(x, t)$, уравнения (19), имеюших одинаковую норму: $\|\psi(\cdot, t)\|=\|\phi(\cdot, t)\|=1 / \sqrt{\alpha}$. Их произведение $\psi(x, t) \phi^{*}(\xi, t)$ будет удовлетворять уравнению

$$
\begin{aligned}
i \hbar \frac{\partial}{\partial t} \psi(x) \phi^{*}(\xi) & =\int d y \mathcal{H}_{\psi}(x, y) \psi(y) \phi^{*}(\xi)-\int d y \psi(x) \phi^{*}(y) \mathcal{H}_{\phi}(y, \xi), \\
\mathcal{H}_{\psi}(x, y) & =\left(-\frac{\hbar^{2}}{2 m} \Delta_{x}+U(x)+\int d \xi V(x-\xi) \psi^{*}(\xi) \psi(\xi)\right) \delta(x-y),
\end{aligned}
$$

здесь и всюду ниже аргумент $t$ опушен. $\mathcal{H}_{\phi}(x, y)$ определяется аналогичным образом. Из (20) следует, что оператор $\hat{u}$, действуюший по формуле

$$
\hat{u} f(x)=\alpha(2 \pi \hbar)^{-\frac{3}{2}} \psi(x) \int d \xi \phi^{*}(\xi) f(\xi), \quad f \in L^{2}\left(\mathbb{R}^{3}\right),
$$

удовлетворяет уравнению

$$
\frac{\partial}{\partial t} \hat{u}=-\frac{i}{\hbar}\left(\widehat{H}_{\psi} \hat{u}-\hat{u} \widehat{H}_{\phi}\right)
$$

где $\widehat{H}_{\psi}$ и $\widehat{H}_{\phi}$ - операторы, задаваемые интегральными ядрами $\mathcal{H}_{\psi}(x, y)$ и $\mathcal{H}_{\phi}(x, y)$, соответственно. Оператор $\hat{u}$ можно записать как вейлевский $\hbar$-псевдодифференциальный оператор,

$$
\hat{u}=u\left(\frac{1}{x+3} \frac{3}{2},-i \hbar \frac{\stackrel{2}{\partial}}{\partial x}\right)
$$

(индексы над операторами в (23) обозначают порядок их действия [5]), с символом

$$
\begin{aligned}
& u(x, p)=\frac{\alpha}{(2 \pi \hbar)^{\frac{3}{2}}} \int d q e^{-\frac{i}{\hbar} p q} \psi\left(x+\frac{q}{2}\right) \phi^{*}\left(x-\frac{q}{2}\right), \\
& \hat{u} f(x)=\frac{1}{(2 \pi \hbar)^{\frac{3}{2}}} \iint d p d \xi u\left(\frac{x+\xi}{2}, p\right) e^{\frac{i}{\hbar} p(x-\xi)} f(\xi) .
\end{aligned}
$$

Уравнение для символа имеет вид

$$
\frac{\partial}{\partial t} u(x, p)=-\frac{i}{\hbar}\left(H_{\psi}(x, p) * u(x, p)-u(x, p) * H_{\phi}(x, p)\right),
$$

где “*” - умножение символов (скрученное произведение [6]), индуцированное операцией умножения операторов, $H_{\psi}(x, p)$ и $H_{\phi}(x, p)$ - вейлевские символы операторов $\widehat{H}_{\psi}$ и $\widehat{H}_{\phi}$, соответственно:

$$
H_{\psi}(x, p)=\frac{p^{2}}{2 m}+U(x)+\int d \xi V(x-\xi)|\psi(\xi)|^{2}
$$

и аналогично для $H_{\phi}(x, p)$. 
Несложно проверить, что оператор $\hat{\bar{u}}$, соответствующий комплексно сопряженному символу $\bar{u}(x, p)$, действует согласно формуле

$$
\widehat{\bar{u}} f(x)=\alpha(2 \pi \hbar)^{-\frac{3}{2}} \phi(x) \int d \xi \psi^{*}(\xi) f(\xi), \quad f \in L^{2}\left(\mathbb{R}^{3}\right) .
$$

Из формул (21), (25) следует, что с точностью до постоянного множителя скрученные произведения символов $u(x, p) * \bar{u}(x, p)$ и $\bar{u}(x, p) * u(x, p)$ являются символами операторов проектирования $\hat{\pi}_{\psi}$ и $\hat{\pi}_{\phi}$ на состояния $\sqrt{\alpha} \psi$ и $\sqrt{\alpha} \phi$, соответственно:

$$
\begin{aligned}
& (u * \bar{u})(x, p)=\alpha^{-1}(2 \pi \hbar)^{-3} \operatorname{smb} \hat{\pi}_{\psi}(x, p), \\
& (\bar{u} * u)(x, p)=\alpha^{-1}(2 \pi \hbar)^{-3} \operatorname{smb} \hat{\pi}_{\phi}(x, p) .
\end{aligned}
$$

Учитывая эти формулы, а также формулу для следа псевдодифференциального оператора

$$
\operatorname{Tr} \widehat{A}=\frac{1}{(2 \pi \hbar)^{3}} \iint d x d p(\operatorname{smb} \widehat{A})(x, p),
$$

символы $H_{\psi}$ и $H_{\phi}$ можно выразить через $u$ и $\bar{u}$ :

$$
\begin{aligned}
& H_{\psi}(x, p)=\frac{p^{2}}{2 m}+U(x)+\iint d \xi d \eta V(x-\xi) *(u * \bar{u})(\xi, \eta), \\
& H_{\phi}(x, p)=\frac{p^{2}}{2 m}+U(x)+\iint d \xi d \eta V(x-\xi) *(\bar{u} * u)(\xi, \eta)
\end{aligned}
$$

(скрученные произведения в интегральных членах вычисляются по переменным $\xi$ и $\eta$ ). После подстановки (28) и (29) в (24) и циклической перестановки сомножителей под знаком интегрирования уравнение для $u(x, p)$ примет вид

$$
\begin{aligned}
\frac{\partial}{\partial t} u(x, p)= & -\left\{\frac{p^{2}}{2 m}+U(x), u(x, p)\right\}_{\hbar}- \\
& -\iint d \xi d \eta \bar{u}(\xi, \eta) *\{V(x-\xi), u(\xi, \eta) u(x, p)\}_{\hbar},
\end{aligned}
$$

где, как и ранее, у функций $u$ и $\bar{u}$ опушен аргумент $t ;\{a, b\}_{\hbar}:=(i / \hbar)(a * b-b * a)-$ квантовые скобки Пуассона; скрученные произведения вычисляются как по переменным $x, p$, так и по переменным $\xi, \eta$. Условия конечности норм $\|\psi\|,\|\phi\|<\infty$ индуцируют условие на $u$

$$
\iint d x d p|u(x, p)|^{2}<\infty
$$

которое получается из (26), (27) с учетом формулы

$$
\iint d x d p(a * b)(x, p)=\iint d x d p a(x, p) b(x, p) .
$$

Отметим, что уравнение (30) возникало в работе [7] при построении асимптотических решений многочастичного уравнения для квантовой функции плотности системы большого числа различных частиц - уравнения Вигнера.

Чтобы перейти от квантового уравнения для $u(x, p)$ к его классическому аналогу, необходимо заменить скрученное произведение на обычное, а квантовые скобки Пуассона $\{\cdot, \cdot\}_{\hbar}$ - на классические $\{\cdot, \cdot\}$. Несложно видеть, что получающееся в результате этого классическое уравнение совпадает с (3). 


\section{4. ОБОБШЕНИЕ УРАВНЕНИЯ ДЛЯ АМПЛИТУДЫ}

Представленный вывод уравнения (3), в отличие от вывода из вторично квантованного уравнения (1), можно перенести на интересуюший нас более обший случай системы большого числа частиц с электромагнитным взаимодействием, в которой имеются несколько различных сортов частиц. Заметим, что хотя в представленном выше рассуждении мы исходили из вторично квантованного уравнения Шредингера для нерелятивистких бозонов, окончательное классическое $(\hbar=0)$ уравнение $(3)$ оказывается применимым для любой классической системы большого числа одинаковых частиц независимо от типа статистики, которой они подчиняются в квантовой механике. Имея в виду этот факт ограничим наше рассмотрение (формальным) предположением, что все частишы в системе являются нерелятивисткими бесспиновыми бозонами.

Вместо уравнения (19) естественно взять уравнения вида

$$
i \hbar \frac{\partial}{\partial t} \psi_{a}(x, t)=\left(\frac{1}{2 m_{a}} \sum_{j=1}^{3}\left(-i \hbar \frac{\partial}{\partial x_{j}}-\frac{e_{a}}{c} A_{j}(x, t)\right)^{2}+e_{a} \Phi(x, t)\right) \psi_{a}(x, t),
$$

где $t \in \mathbb{R}, \quad x \in \mathbb{R}^{3}, \quad \psi_{a}(\cdot, t) \in L^{2}\left(\mathbb{R}_{x}^{3}\right)$, индекс $a=1, \ldots, a_{0}$ нумерует различные типы частиц, $e_{a}$ и $m_{a}$ - заряд и масса частицы сорта $a$, соответственно, $c$ - скорость света, $\Phi(x, t)$ и $A_{j}(x, t)$ - потенциалы электромагнитного поля. Уравнения для $\Phi$ и $A_{j}$ связаны самосогласованным образом с волновыми функциями $\psi_{a}$. Удобнее всего записать их в кулоновской калибровке

$$
\sum_{j=1}^{3} \frac{\partial}{\partial x_{j}} A_{j}(x, t)=0 .
$$

Кроме того, мы будем предполагать, что потенциалы электромагнитного поля являются быстро убывающими функциями на пространственной бесконечности. Уравнения для $\Phi$ и $A_{j}$ в данной калибровке имеют вид

$$
\begin{gathered}
\Delta \Phi(x, t)=-4 \pi \sum_{a=1}^{a_{0}} e_{a}\left|\psi_{a}(x, t)\right|^{2} \\
\left(\frac{1}{c^{2}} \frac{\partial^{2}}{\partial t^{2}}-\Delta\right) A_{j}(x, t)-\frac{1}{c} \frac{\partial^{2}}{\partial x_{j} \partial t} \Phi(x, t)= \\
=\frac{4 \pi}{c} \sum_{a=1}^{a_{0}} \frac{e_{a}}{m_{a}}\left(-\frac{i \hbar}{2}\left(\psi_{a}^{*}(x, t) \frac{\partial \psi_{a}(x, t)}{\partial x_{j}}-\psi_{a}(x, t) \frac{\partial \psi_{a}^{*}(x, t)}{\partial x_{j}}\right)-\right. \\
\left.-\frac{e_{a}}{c} A_{j}(x, t) \psi_{a}^{*}(x, t) \psi_{a}(x, t)\right) .
\end{gathered}
$$

Отметим, что система уравнений (32)-(35) может быть получена из условия стационарности функционала действия (для сокрашения записи аргументы $(x, t)$ опускаем)

$$
\begin{aligned}
S= & \iint d t d x \sum_{a=1}^{a_{0}}\left\{i \psi_{a}^{*}\left(\hbar \partial_{t}+i e_{a} \Phi\right) \psi_{a}-\frac{1}{2 m_{a}}\left|\left(\hbar \partial_{j}-\frac{i e_{a}}{c} A_{j}\right) \psi_{a}\right|^{2}\right\}+ \\
& +\frac{1}{4 \pi} \iint d t d x\left\{\frac{1}{2}\left(\partial_{j} \Phi+\frac{1}{c} \partial_{t} A_{j}\right)^{2}-\frac{1}{4}\left(\partial_{j} A_{l}-\partial_{l} A_{j}\right)^{2}\right\}
\end{aligned}
$$


на конфигурациях, удовлетворяющих условию калибровки (33) (вариации $\psi_{a}$ и $\psi_{a}^{*}$ при этом рассматриваются как независимые). Здесь $\partial_{t} \equiv \partial / \partial t, \partial_{j} \equiv \partial / \partial x_{j}$, по индексам $j$ и $l$ подразумевается суммирование. Функционал подобного вида в случае $a_{0}=2, e_{1}=$ $-e_{2}$ рассматривался в работе [8].

Поступим теперь с системой уравнений (32), (34), (35) подобно тому, как мы поступили с уравнением (19) в предыдущем разделе. Пусть $\psi_{a}, \Phi^{(\psi)}, A_{j}^{(\psi)}$ и $\phi_{a}, \Phi^{(\phi)}, A_{j}^{(\phi)}-$ какие-нибудь два решения данной системы, причем $\psi_{a}$ и $\phi_{a}$ имеют одинаковые нормы, $\left\|\psi_{a}(\cdot, t)\right\|=\left\|\phi_{a}(\cdot, t)\right\|=1 / \sqrt{\alpha_{a}}$. Тогда набор $u_{a}(x, p, t)$,

$$
u_{a}(x, p, t)=\frac{\alpha_{a}}{(2 \pi \hbar)^{\frac{3}{2}}} \int d q e^{-\frac{i}{\hbar} p q} \psi_{a}\left(x+\frac{q}{2}, t\right) \phi_{a}^{*}\left(x-\frac{q}{2}, t\right),
$$

будет удовлетворять уравнениям

$$
\frac{\partial}{\partial t} u_{a}(x, p)=-\frac{i}{\hbar}\left(H_{a}^{(\psi)}(x, p) * u_{a}(x, p)-u_{a}(x, p) * H_{a}^{(\phi)}(x, p)\right),
$$

где, а также всюду ниже опущен аргумент $t$,

$$
H_{a}^{(\psi)}(x, p)=\sum_{a=1}^{a_{0}}\left(\frac{1}{2 m_{a}} \sum_{j=1}^{3}\left(p_{j}-\frac{e_{a}}{c} A_{j}^{(\psi)}(x)\right)^{2}+e_{a} \Phi^{(\psi)}(x)\right)
$$

$H_{a}^{(\phi)}$ определяются так же, как $H_{a}^{(\psi)}$, с заменой индекса $\psi$ на $\phi$. Гамильтонианы $H_{a}^{(\psi)}$ и $H_{a}^{(\phi)}$ можно выразить через произведения $\left(u_{a} * \bar{u}_{a}\right)(x, p)$ и $\left(\bar{u}_{a} * u_{a}\right)(x, p)$. Чтобы в этом убедиться, достаточно показать, что правые части уравнений (34), (35) могут быть выражены через эти произведения. В самом деле, воспользовавшись формулами вида (26), (27), можно получить

$$
\begin{gathered}
\psi_{a}^{*}(x) \psi_{a}(x)=\int d p\left(u_{a} * \bar{u}_{a}\right)(x, p), \quad \phi_{a}^{*}(x) \phi_{a}(x)=\int d p\left(\bar{u}_{a} * u_{a}\right)(x, p), \\
-i \hbar\left(\psi_{a}^{*}(x) \frac{\partial \psi_{a}(x)}{\partial x_{j}}-\psi_{a}(x) \frac{\partial \psi_{a}^{*}(x)}{\partial x_{j}}\right)= \\
=-i \hbar \int d \xi \frac{\partial \delta(x-\xi)}{\partial x_{j}}\left(\psi_{a}^{*}(x) \psi_{a}(\xi)-\psi_{a}(x) \psi_{a}^{*}(\xi)\right)= \\
=\int d p \operatorname{smb}\left(-i \hbar \frac{\partial}{\partial x_{j}} \hat{u}_{a} \hat{\bar{u}}_{a}+\hat{u}_{a} \hat{\bar{u}}_{a}\left(-i \hbar \frac{\partial}{\partial x_{j}}\right)\right)(x, p)= \\
=\int d p\left(p_{j} * u_{a}(x, p) * \bar{u}_{a}(x, p)+u_{a}(x, p) * \bar{u}_{a}(x, p) * p_{j}\right), \\
-i \hbar\left(\phi_{a}^{*}(x) \frac{\partial \phi_{a}(x)}{\partial x_{j}}-\phi_{a}(x) \frac{\partial \phi_{a}^{*}(x)}{\partial x_{j}}\right)= \\
=\int d p\left(p_{j} * \bar{u}_{a}(x, p) * u_{a}(x, p)+\bar{u}_{a}(x, p) * u_{a}(x, p) * p_{j}\right) .
\end{gathered}
$$


Перейдем теперь в уравнении (36) к классическому пределу (всюду далее в обозначениях $u_{a}, H_{a}^{(\psi)}$ и $H_{a}^{(\phi)}$ для сокрашения записи будем опускать аргументы $\left.(x, p)\right)$. С этой целью его удобно предварительно переписать в виде

$$
\begin{aligned}
\frac{\partial}{\partial t} u_{a}= & -\frac{i}{\hbar}\left(\frac{H_{a}^{(\psi)}+H_{a}^{(\phi)}}{2} * u_{a}-u_{a} * \frac{H_{a}^{(\psi)}+H_{a}^{(\phi)}}{2}\right)- \\
& -\frac{i}{2}\left(\frac{H_{a}^{(\psi)}-H_{a}^{(\phi)}}{\hbar} * u_{a}+u_{a} * \frac{H_{a}^{(\psi)}-H_{a}^{(\phi)}}{\hbar}\right)
\end{aligned}
$$

и затем представить разность $H_{a}^{(\psi)}-H_{a}^{(\phi)}$ в виде

$$
\begin{aligned}
H_{a}^{(\psi)}-H_{a}^{(\phi)}= & -\frac{e_{a}}{m_{a} c} \sum_{j=1}^{3}\left(p_{j}-\frac{e_{a}}{c} \frac{A_{j}^{(\psi)}(x)+A_{j}^{(\phi)}(x)}{2}\right)\left(A_{j}^{(\psi)}(x)-A_{j}^{(\phi)}(x)\right)+ \\
& +e_{a}\left(\Phi^{(\psi)}(x)-\Phi^{(\phi)}(x)\right) .
\end{aligned}
$$

Уравнения для $\left(\Phi^{(\psi)}(x)-\Phi^{(\phi)}(x)\right) / \hbar$ и $\left(A_{j}^{(\psi)}(x)-A_{j}^{(\phi)}(x)\right) / \hbar$ имеют вид

$$
\begin{gathered}
\Delta \frac{\Phi^{(\psi)}(x)-\Phi^{(\phi)}(x)}{\hbar}=-4 \pi \sum_{a=1}^{a_{0}} e_{a} \int d p \frac{u_{a} * \bar{u}_{a}-\bar{u}_{a} * u_{a}}{\hbar} \\
\left(\frac{1}{c^{2}} \frac{\partial^{2}}{\partial t^{2}}-\Delta\right) \frac{A_{j}^{(\psi)}(x)-A_{j}^{(\phi)}(x)}{\hbar}-\frac{1}{c} \frac{\partial^{2}}{\partial x_{j} \partial t} \frac{\Phi^{(\psi)}(x)-\Phi^{(\phi)}(x)}{\hbar}= \\
=\frac{4 \pi}{c} \sum_{a=1}^{a_{0}} \frac{e_{a}}{m_{a}}\left(\int d p p_{j} * \frac{u_{a} * \bar{u}_{a}-\bar{u}_{a} * u_{a}}{\hbar}-\right. \\
-\frac{e_{a}}{c} \frac{A_{j}^{(\psi)}(x)-A_{j}^{(\phi)}(x)}{\hbar} \int d p u_{a} * \bar{u}_{a}- \\
\left.-\frac{e_{a}}{c} \frac{A_{j}^{(\psi)}(x)+A_{j}^{(\phi)}(x)}{2} \int d p \frac{u_{a} * \bar{u}_{a}-\bar{u}_{a} * u_{a}}{\hbar}\right) .
\end{gathered}
$$

Устремляя $\hbar \rightarrow 0$ и предполагая, что

$$
\begin{aligned}
\Phi^{(\psi)}(x), \Phi^{(\phi)}(x) & \rightarrow \Phi(x), & A_{j}^{(\psi)}(x), A_{j}^{(\phi)}(x) & \rightarrow A_{j}(x), \\
\frac{\Phi^{(\psi)}(x)-\Phi^{(\phi)}(x)}{\hbar} & \rightarrow \widetilde{\Phi}(x), & \frac{A_{j}^{(\psi)}(x)-A_{j}^{(\phi)}(x)}{\hbar} & \rightarrow \tilde{A}_{j}(x),
\end{aligned}
$$

получим в итоге систему уравнений (9)-(13). Кроме того, условие кулоновской калибровки (33) дает в классическом пределе условия (14) для $A_{j}$ и $\tilde{A}_{j}$. 


\section{5. ГАМИЛЬТОНОВОСТЬ УРАВНЕНИЙ И ИХ СВЯЗЬ С АСИМПТОТИЧЕСКИМИ РЕШЕНИЯМИ ДЛЯ $\bar{N} \rightarrow \infty$}

Перед тем как обсудить полученную систему уравнений (9)-(14), выясним, какой смысл имеют квантовые уравнения (32)-(35) с точки зрения асимптотики вторично квантованных уравнений. Указанные уравнения являются аналогом уравнения (19), которое играет, в свою очередь, роль системы Гамильтона при построении асимптотики уравнения Шредингера (18) по параметру $\varepsilon \rightarrow 0$. Если в качестве асимптотических решений этого уравнения взять решения, функционалы которых в представлении Шредингера сосредоточены в окрестности точки $\sim \sqrt{\varepsilon}$ (асимптотики типа волновых пакетов, построенные в работе [9]), то окажется, что среднее $\bar{N}$ от оператора числа частиц $\widehat{N}=\int d x \psi^{+}(x) \psi^{-}(x)$ по таким состояниям будет порядка $1 / \varepsilon$. Это означает, что в сушности асимптотика по параметру $\varepsilon \rightarrow 0$ - это асимптотика по среднему числу частиц $\bar{N} \rightarrow \infty$. Отметим, что наличие малого параметра $\varepsilon \sim 1 / \bar{N}$ в гамильтониане уравнения (18) приводит к тому, что среднее значение энергии системы растет линейно по $\bar{N}$ при $\bar{N} \rightarrow \infty$.

Систему уравнений (32)-(35) также можно свести к системе Гамильтона. Это делается следуюшим образом. Прежде всего необходимо отметить, что данная система уравнений эквивалентна системе

$$
\begin{gathered}
i \frac{\partial}{\partial t} \psi_{a}(x)=\frac{\delta}{\delta \psi_{a}^{*}(x)} K\left(\psi^{*}, \psi, \dot{A}, A, \Phi\right), \\
\frac{\delta}{\delta \Phi(x)} K\left(\psi^{*}, \psi, \dot{A}, A, \Phi\right)=0, \\
\left(\frac{d}{d t} \frac{\delta}{\delta \dot{A}_{j}(x)}-\frac{\delta}{\delta A_{j}(x)}\right) K\left(\psi^{*}, \psi, \dot{A}, A, \Phi\right)=0, \\
\sum_{j=1}^{3} \frac{\partial}{\partial x_{j}} A_{j}(x)=0,
\end{gathered}
$$

где

$$
\begin{aligned}
K= & \frac{1}{\hbar} \sum_{a=1}^{a_{0}} \int d x\left(\psi_{a}^{*}(x) \frac{1}{2 m_{a}} \sum_{j=1}^{3}\left(-i \hbar \frac{\partial}{\partial x_{j}}-\frac{e_{a}}{c} A_{j}(x)\right)^{2} \psi_{a}(x)+e_{a} \Phi(x) \psi_{a}^{*}(x) \psi_{a}(x)\right)- \\
& -\frac{1}{4 \pi \hbar} \int d x\left(\frac{1}{2} \sum_{j=1}^{3}\left(\frac{1}{c} \dot{A}_{j}(x)+\frac{\partial \Phi(x)}{\partial x_{j}}\right)^{2}-\frac{1}{4} \sum_{j, l=1}^{3}\left(\frac{\partial A_{l}(x)}{\partial x_{j}}-\frac{\partial A_{j}(x)}{\partial x_{l}}\right)^{2}\right)
\end{aligned}
$$

и для компактности записи опускается аргумент $t$. Функционал $K$ играет роль гамильтониана по переменным $\psi_{a}^{*}(x)$ и $\psi_{a}(x)$ и роль функции Лагранжа по потенциалам поля $\Phi(x), A_{j}(x)$. В силу вырожденности $K$ как функции Лагранжа невозможно представить всю систему уравнений в гамильтоновой форме, просто взяв преобразование Лежандра по скоростям переменных электромагнитного поля. В связи с этим приходится поступить следуюшим образом. Сначала с помошью уравнения (37) исключаются циклические переменные $\Phi(x), \Phi(x)=\Phi\left[\psi^{*}, \psi, \dot{A}, A\right](x)$. Остаюшиеся при этом уравнения снова 
могут быть записаны частично в гамильтоновой, а частично в лагранжевой форме, причем вместо $K$ следует взять

$$
K^{\prime}\left(\psi^{*}, \psi, \dot{A}, A\right)=K\left(\psi^{*}, \psi, \dot{A}, A, \Phi\left[\psi^{*}, \psi, \dot{A}, A\right]\right) .
$$

Далее уравнения с $K^{\prime}$ ограничиваются на конфигурации, удовлетворяющие условию кулоновской калибровки (38). Для этого вектор-потенциал удобно представить в виде

$$
A_{j}(x)=\int^{\prime} d k \sum_{s=1}^{2} e_{s j}(k)\left(a_{s}(k) e^{i k x}+a_{s}^{*}(k) e^{-i k x}\right),
$$

где $k \in \mathbb{R}^{3}$, штрих у интеграла означает, что интегрирование ведется по полупространству (задаваемому, скажем, неравенством $\left.k_{1}>0\right),\left\{e_{s}(k)\right\}, s=1,2,-$ произвольная пара ортонормированных векторов, ортогональных $k$,

$$
\begin{gathered}
\sum_{j=1}^{3} e_{s j}(k) e_{r j}(k)=\delta_{r s}, \quad \sum_{j=1}^{3} e_{s j}(k) k_{j}=0 \\
\sum_{s=1}^{2} e_{s i}(k) e_{s j}(k)+\frac{k_{i} k_{j}}{|k|^{2}}=\delta_{i j} .
\end{gathered}
$$

Действительные и мнимые части функций $a_{s}$, умноженные на $\sqrt{2}\left(a_{s}(k)=\left(q_{s}(k)+\right.\right.$ $\left.i \xi_{s}(k)\right) / \sqrt{2}$ ), можно воспринимать как новые координаты. Уравнения для них будут иметь вид

$$
\begin{aligned}
& \left(\frac{d}{d t} \frac{\delta}{\delta \dot{q}_{s}(k)}-\frac{\delta}{\delta q_{s}(k)}\right) K^{\prime \prime}\left(\psi^{*}, \psi, \dot{q}, q, \dot{\xi}, \xi\right)=0 \\
& \left(\frac{d}{d t} \frac{\delta}{\delta \dot{\xi}_{s}(k)}-\frac{\delta}{\delta \xi_{s}(k)}\right) K^{\prime \prime}\left(\psi^{*}, \psi, \dot{q}, q, \dot{\xi}, \xi\right)=0
\end{aligned}
$$

где

$$
K^{\prime \prime}=K^{\prime}\left(\psi^{*}, \psi, B[\dot{q}, \dot{\xi}], A[q, \xi]\right),
$$

$(A[q, \xi])_{j}(x)$ - правая часть формулы $(39),(B[\dot{q}, \dot{\xi}])_{j}(x)$ - правая часть $(39)$, продиффференцированная по $t$. Уравнение для $\psi_{a}(x)$ можно записать в виде

$$
i \frac{\partial}{\partial t} \psi_{a}(x)=\frac{\delta}{\delta \psi_{a}^{*}(x)} K^{\prime \prime}\left(\psi^{*}, \psi, \dot{q}, q, \dot{\xi}, \xi\right) .
$$

Отметим, что сохранение лагранжевости по одной части переменных $\left(q_{s}(k), \xi_{s}(k)\right)$ и гамильтоновости по другой $\left(\psi_{a}(x), \psi_{a}^{*}(x)\right)$ обусловлено тем, что при ограничении функционала $K^{\prime}\left(\psi^{*}, \psi, \dot{A}, A\right)$ на конфигурации вида (39) он распадается на сумму двух слагаемых:

$$
K^{\prime} \rightarrow H_{0}\left(\psi^{*}, \psi, A\right)+L(\dot{A}, A)
$$


первое из которых, содержашее (комплексные) гамильтоновы переменные $\psi$ и $\psi^{*}$, не содержит $\dot{A}$, а второе, содержашее $\dot{A}$, не содержит $\psi$ и $\psi^{*}$. Функционал $K^{\prime \prime}$ (рассматриваемый как аналог функции Лагранжа) в отличие от исходного функционала $K$ не вырожден по скоростям. Соответствующий ему гамильтониан $H^{\prime \prime}$ определяется однозначно с помощью преобразования Лежандра и имеет вид

$$
\begin{aligned}
H^{\prime \prime}= & \frac{1}{\hbar} \sum_{a=1}^{a_{0}} \int d x \psi_{a}^{*}(x) \frac{1}{2 m_{a}} \sum_{j=1}^{3}\left(-i \hbar \frac{\partial}{\partial x_{j}}-\frac{e_{a}}{c}\left(\tilde{A}\left[a, a^{*}\right]\right)_{j}(x)\right)^{2} \psi_{a}(x)+ \\
& +\frac{1}{2 \hbar} \sum_{a, b=1}^{a_{0}} \iint d x d y \psi_{a}^{*}(x) \psi_{b}^{*}(y) \frac{e_{a} e_{b}}{|x-y|} \psi_{b}(y) \psi_{a}(x)+ \\
& +\sum_{s=1}^{2} \int^{\prime} d k\left(c^{2} \frac{4 \pi \hbar}{(2 \pi)^{3}} b_{s}^{*}(k) b_{s}(k)+\frac{(2 \pi)^{3}}{4 \pi \hbar}|k|^{2} a_{s}^{*}(k) a_{s}(k)\right),
\end{aligned}
$$

где $a_{s}(k)=\left(q_{s}(k)+i \xi_{s}(k)\right) / \sqrt{2}, b_{s}(k)=\left(p_{s}(k)+i \eta_{s}(k)\right) / \sqrt{2}, p_{s}(k)$ и $\eta_{s}(k)$ - импульсы, канонически сопряженные к переменным $q_{s}(k)$ и $\xi_{s}(k)$, через $\left(\tilde{A}\left[a, a^{*}\right]\right)_{j}(x)$ обозначена правая часть формулы (39). Отметим, что нетривиальные скобки Пуассона между переменными, фигурирующими в правой части (40), равны

$$
\left\langle\psi_{a}(x), \psi_{b}^{*}(y)\right\rangle=i \delta_{a b} \delta(x-y), \quad\left\langle b_{s}(k), a_{r}^{*}(q)\right\rangle=\delta_{r s} \delta(k-q) .
$$

Остальные скобки равны нулю. Здесь мы использовали обозначение для ( "вторичных" ) скобок Пуассона $\langle\cdot, \cdot\rangle$, чтобы не путать их с обычными скобками Пуассона $\{\cdot, \cdot\}$, используемыми в классической механике.

ЗАмЕЧАниЕ. Гамильтониан $H^{\prime \prime}$ позволяет найти энергию $E$ всей системы: $E=\hbar H^{\prime \prime}$. На статических конфигурациях

$$
\dot{a}_{s}(k)=\frac{\delta H^{\prime \prime}}{\delta b_{s}^{*}(k)}=c^{2} \frac{4 \pi \hbar}{(2 \pi)^{3}} b_{s}(k)=0,
$$

и, следовательно, $b_{s}(k)=b_{s}^{*}(k)=0$. Учитывая, что

$$
\begin{aligned}
\sum_{s=1}^{2} \int^{\prime} d k|k|^{2} a_{s}^{*}(k) a_{s}(k) & =\frac{1}{2(2 \pi)^{3}} \int d x\left(\partial_{i} A_{j}(x)\right) \partial_{i} A_{j}(x)= \\
& =\frac{1}{2(2 \pi)^{3}} \int d x H_{i}(x) H_{i}(x),
\end{aligned}
$$

где $\partial_{i}=\partial / \partial x_{i}, H_{i}(x)=\varepsilon_{i j l} \partial_{j} A_{l}, \varepsilon_{i j l}$ - абсолютно антисимметричный тензор, по повторяющимся индексам подразумевается суммирование, получим выражение для функционала энергии в виде

$$
\begin{aligned}
E= & \sum_{a=1}^{a_{0}} \int d x \frac{1}{2 m_{a}}\left|\left(\hbar \partial_{j}-\frac{i e_{a}}{c} A_{j}(x)\right) \psi_{a}(x)\right|^{2}+ \\
& +\sum_{a, b=1}^{a_{0}} \iint d x d y \frac{e_{a} e_{b}}{|x-y|}\left|\psi_{a}(x)\right|^{2}\left|\psi_{b}(x)\right|^{2}+\frac{1}{8 \pi} \int d x H_{j}^{2}(x)
\end{aligned}
$$


(по индексу $j$ подразумевается суммирование). Пусть рассматриваемая система представляет собой двухкомпонентную плазму $\left(a_{0}=2\right)$ такую, что $e_{1}=-e_{2}=e$. Предположим, далее, что имеет место сильная дебаевская экранировка. Это позволяет заменить в функционале энергии $E$ функции $e^{2} /|x-y|$, стояшие в подынтегральных выражениях, на $g \delta(x-y)$, где $g$ - константа связи. В итоге функционал энергии $E$ примет вид

$$
\begin{aligned}
E= & \int d x\left[\frac{1}{2 \mu}\left\{\cos ^{2} \alpha\left|\left(\hbar \partial_{j}-\frac{i e}{c} A_{j}\right) \psi_{1}\right|^{2}+\sin ^{2} \alpha\left|\left(\hbar \partial_{j}+\frac{i e}{c} A_{j}\right) \psi_{2}\right|^{2}\right\}+\right. \\
& \left.+\frac{1}{8 \pi} H_{j}^{2}+g\left(\psi_{2}^{*} \psi_{2}-\psi_{1}^{*} \psi_{1}\right)^{2}\right]
\end{aligned}
$$

где $\mu=m_{1} \sin ^{2} \alpha=m_{2} \cos ^{2} \alpha-$ приведенная масса. Такой функционал рассматривался в работе [8].

Задание четырех функций $q_{s}(k), \xi_{s}(k), p_{s}(k)$ и $\eta_{s}(k)$ ( $s$ фиксировано) на полупространстве эквивалентно заданию двух функций $\mathcal{Q}_{s}(k)$ и $\mathcal{P}_{s}(k)$ на всем пространстве $k \in \mathbb{R}^{3}$. Подберем такое преобразование $q_{s}, \xi_{s}, p_{s}, \eta_{s} \rightarrow \mathcal{Q}_{s}, \mathcal{P}_{s}$, чтобы в терминах переменных $\mathcal{A}_{s}(k)=\left(\mathcal{Q}_{s}(k)+i \mathcal{P}_{s}(k)\right) / \sqrt{2}$ вектор-потенциал $A_{j}(x)$ принял вид

$$
A_{j}(x)=\sum_{s=1}^{2} \int d k f(k) e_{s j}(k)\left(e^{i k x} \mathcal{A}_{s}(k)+e^{-i k x} \mathcal{A}_{s}^{*}(k)\right)
$$

а не исчезающая при $\psi=0$ часть гамильтониана $H^{\prime \prime}$ приняла бы вид

$$
\left.H^{\prime \prime}\right|_{\psi=0}=\sum_{s=1}^{2} \int d k g(k) \mathcal{A}_{s}^{*}(k) \mathcal{A}_{s}(k)
$$

где $f(k)$ и $g(k)$ - некоторые функции. Оказывается, что эти два требования вместе с требованиями каноничности преобразования,

$$
\left\langle\mathcal{A}_{s}(k), \mathcal{A}_{r}^{*}(q)\right\rangle=i \delta_{r s} \delta(k-q), \quad\left\langle\mathcal{A}_{s}(k), \mathcal{A}_{r}(q)\right\rangle=0
$$

однозначно определяют функции $f(k)$ и $g(k)$ :

$$
f(k)=\frac{1}{2 \pi} \sqrt{\frac{\hbar c}{|k|}}, \quad g(k)=c|k| .
$$

Само же каноническое преобразование дается формулами

$$
\begin{aligned}
& a_{s}(k)=\frac{1}{2 \pi} \sqrt{\frac{\hbar c}{|k|}}\left(\mathcal{A}_{s}(k)+e_{s j}(k) \sum_{r=1}^{2} e_{r j}(-k) \mathcal{A}_{r}^{*}(-k)\right), \\
& b_{s}(k)=i \pi \sqrt{\frac{\hbar c}{|k|}}\left(-\mathcal{A}_{s}(k)+e_{s j}(k) \sum_{r=1}^{2} e_{r j}(-k) \mathcal{A}_{r}^{*}(-k)\right) .
\end{aligned}
$$


Обозначим гамильтониан $H^{\prime \prime}$, записанный в терминах переменных $\psi_{a}(x), \psi_{a}^{*}(x)$, $\mathcal{A}_{s}(k)$ и $\mathcal{A}_{s}^{*}(k)$, через $H=H\left(\psi^{*}, \psi, \mathcal{A}^{*}, \mathcal{A}\right)$. Представленное рассуждение означает, что систему уравнений (32)-(35) можно воспринимать как систему, эквивалентную системе Гамильтона, соответствующую предельному переходу $\varepsilon \rightarrow 0$ в уравнении Шредингера с гамильтонианом

$$
\widehat{H}_{\varepsilon}=\frac{1}{\varepsilon} H\left(\sqrt{\varepsilon} \psi^{+}, \sqrt{\varepsilon}{ }^{1}-, \sqrt{\varepsilon} \stackrel{2}{\mathcal{A}}^{+}, \sqrt{\varepsilon} \stackrel{1}{\mathcal{A}}^{-}\right),
$$

где $\psi^{ \pm}$- бозевские операторы рождения-уничтожения, соответствуюшие переменным $\psi^{*}$ и $\psi, \mathcal{A}^{ \pm}$- бозевские операторы рождения-уничтожения, соответствуюшие переменным $\mathcal{A}^{*}$ и $\mathcal{A}$. Асимптотику по параметру $\varepsilon$ можно трактовать как асимптотику по большому среднему числу частиц $\bar{N}_{a}$ каждого сорта $a, \bar{N}_{a} \sim\left\|\psi_{a}\right\|^{2} / \varepsilon \rightarrow \infty$, и числу фотонов $\bar{N}_{0} \sim\|\mathcal{A}\|^{2} / \varepsilon \rightarrow \infty$. Подобный предельный переход имеет место в теории излучения Гайтлера [10]. Отметим, что гамильтониан, аналогичный $\widehat{H}_{1}$, в котором вместо бозевских операторов $\psi^{ \pm}$стоят фермионные операторы рождения-уничтожения [2], возникал в [11].

Представленная схема рассуждений легко переносится на интересуюший нас случай классических уравнений (9)-(14). Несложно проверить, что уравнения (9)-(14) эквивалентны системе уравнений

$$
\begin{gathered}
i \frac{\partial}{\partial t} u_{a}(x, p)=\frac{\delta}{\delta u_{a}^{*}(x, p)} \mathcal{K}\left(u^{*}, u, \dot{A}, A, \Phi, \dot{\tilde{A}}, \tilde{A}, \widetilde{\Phi}\right) \\
\frac{\delta \mathcal{K}}{\delta \Phi(x)}=0, \quad \frac{\delta \mathcal{K}}{\delta \widetilde{\Phi}(x)}=0 \\
\left(\frac{d}{d t} \frac{\delta}{\delta \dot{A}_{j}(x)}-\frac{\delta}{\delta A_{j}(x)}\right) \mathcal{K}=0, \quad\left(\frac{d}{d t} \frac{\delta}{\delta \dot{\tilde{A}}_{j}(x)}-\frac{\delta}{\delta \tilde{A}_{j}(x)}\right) \mathcal{K}=0, \\
\sum_{j=1}^{3} \frac{\partial}{\partial x_{j}} A_{j}(x)=0, \quad \sum_{j=1}^{3} \frac{\partial}{\partial x_{j}} \tilde{A}_{j}(x)=0
\end{gathered}
$$

где

$$
\begin{aligned}
\mathcal{K}\left(u^{*}, u, \dot{A}, A, \Phi, \dot{\tilde{A}}, \tilde{A}, \widetilde{\Phi}\right)=\sum_{a=1}^{a_{0}} \iint d x d p\left(u_{a}^{*}(x, p) \times\right. \\
\quad \times(-i)\left\{\frac{1}{2 m_{a}} \sum_{j=1}^{3}\left(p_{j}-\frac{e_{a}}{c} A_{j}(x)\right)^{2}+e_{a} \Phi(x), u_{a}(x, p)\right\}+ \\
\left.+u_{a}^{*}(x, p)\left[-\frac{e_{a}}{m_{a} c}\left(p_{j}-\frac{e_{a}}{c} A_{j}(x)\right) \tilde{A}_{j}(x)+e_{a} \widetilde{\Phi}(x)\right] u_{a}(x, p)\right)- \\
-\frac{1}{4 \pi} \int d x\left(\sum_{j=1}^{3}\left(\frac{1}{c} \dot{A}_{j}(x)+\frac{\partial \Phi(x)}{\partial x_{j}}\right)\left(\frac{1}{c} \dot{\tilde{A}}_{j}(x)+\frac{\partial \widetilde{\Phi}(x)}{\partial x_{j}}\right)-\right. \\
\left.-\frac{1}{2} \sum_{j, l=1}^{3}\left(\frac{\partial A_{l}(x)}{\partial x_{j}}-\frac{\partial A_{j}(x)}{\partial x_{l}}\right)\left(\frac{\partial \tilde{A}_{l}(x)}{\partial x_{j}}-\frac{\partial \tilde{A}_{j}(x)}{\partial x_{l}}\right)\right) .
\end{aligned}
$$


Гамильтониан, соответствующий $\mathcal{K}$, так же как и в квантовом случае, строится в три этапа. Сначала из уравнений $(42)-(45)$ исключаются циклические переменные $\Phi(x)$ и $\widetilde{\Phi}(x)$. Затем получившиеся уравнения ограничиваются на конфигурации, удовлетворяющие калибровочным условиям (45),

$$
\begin{aligned}
& A_{j}(x)=\int^{\prime} d k \sum_{s=1}^{2} e_{s j}(k)\left(a_{s}(k) e^{i k x}+a_{s}^{*}(k) e^{-i k x}\right), \\
& \tilde{A}_{j}(x)=\int^{\prime} d k \sum_{s=1}^{2} e_{s j}(k)\left(\tilde{a}_{s}(k) e^{i k x}+\tilde{a}_{s}^{*}(k) e^{-i k x}\right)
\end{aligned}
$$

(где $e_{s j}(k)$ имеют тот же смысл, что и в $\left.(39)\right)$, и вычисляется преобразование Лежандра по скоростям

$$
\begin{aligned}
& \sqrt{2} \operatorname{Re} \dot{a}_{s}(k), \sqrt{2} \operatorname{Im} \dot{a}_{s}(k), \sqrt{2} \operatorname{Re} \dot{\tilde{a}}_{s}(k), \sqrt{2} \operatorname{Im} \dot{\tilde{a}}_{s}(k) \rightarrow \\
& \rightarrow \sqrt{2} \operatorname{Re} b_{s}(k), \sqrt{2} \operatorname{Im} b_{s}(k), \sqrt{2} \operatorname{Re} \tilde{b}_{s}(k), \sqrt{2} \operatorname{Im} \tilde{b}_{s}(k) .
\end{aligned}
$$

Наконец, осушествляется каноническое преобразование

$$
\begin{aligned}
& a_{s}(k)=\frac{1}{2 \pi} \sqrt{\frac{c}{|k|}}\left(\mathcal{A}_{s}(k)+e_{s j}(k) \sum_{r=1}^{2} e_{r j}(-k) \mathcal{A}_{r}^{*}(-k)\right), \\
& \tilde{a}_{s}(k)=\frac{1}{2 \pi} \sqrt{\frac{c}{|k|}}\left(\widetilde{\mathcal{A}}_{s}(k)+e_{s j}(k) \sum_{r=1}^{2} e_{r j}(-k) \widetilde{\mathcal{A}}_{r}^{*}(-k)\right), \\
& b_{s}(k)=i \pi \sqrt{\frac{c}{|k|}}\left(-\mathcal{A}_{s}(k)+e_{s j}(k) \sum_{r=1}^{2} e_{r j}(-k) \mathcal{A}_{r}^{*}(-k)\right), \\
& \tilde{b}_{s}(k)=i \pi \sqrt{\frac{c}{|k|}}\left(-\widetilde{\mathcal{A}}_{s}(k)+e_{s j}(k) \sum_{r=1}^{2} e_{r j}(-k) \widetilde{\mathcal{A}}_{r}^{*}(-k)\right),
\end{aligned}
$$

в результате которого вектор-потенциалы $A_{j}(x)$ и $\tilde{A}_{j}(x)$ принимают вид

$$
\begin{aligned}
& A_{j}(x)=\sum_{s=1}^{2} \int d k \frac{1}{2 \pi} \sqrt{\frac{c}{|k|}} e_{s j}(k)\left(e^{i k x} \mathcal{A}_{s}(k)+e^{-i k x} \mathcal{A}_{s}^{*}(k)\right), \\
& \tilde{A}_{j}(x)=\sum_{s=1}^{2} \int d k \frac{1}{2 \pi} \sqrt{\frac{c}{|k|}} e_{s j}(k)\left(e^{i k x} \widetilde{\mathcal{A}}_{s}(k)+e^{-i k x} \widetilde{\mathcal{A}}_{s}^{*}(k)\right),
\end{aligned}
$$

а гамильтониан -

$$
\begin{aligned}
& \mathcal{H}\left(u^{*}, u, \mathcal{A}^{*}, \mathcal{A}, \widetilde{\mathcal{A}}^{*}, \widetilde{\mathcal{A}}\right)=\sum_{a=1}^{a_{0}} \iint d x d p\left(u_{a}^{*}(x, p) \times\right. \\
& \quad \times(-i)\left\{\frac{1}{2 m_{a}} \sum_{j=1}^{3}\left(p_{j}-\frac{e_{a}}{c} A_{j}(x)\right)^{2}+e_{a} \Phi(x), u_{a}(x, p)\right\}+ \\
& \left.\quad+u_{a}^{*}(x, p)\left[-\frac{e_{a}}{m_{a} c}\left(p_{j}-\frac{e_{a}}{c} A_{j}(x)\right) \tilde{A}_{j}(x)+e_{a} \widetilde{\Phi}(x)\right] u_{a}(x, p)\right)+ \\
& \quad+c \sum_{s=1}^{2} \int d k|k|\left(\widetilde{\mathcal{A}}_{s}^{*}(k) \mathcal{A}_{s}(k)+\mathcal{A}_{s}^{*}(k) \widetilde{\mathcal{A}}_{s}(k)\right) .
\end{aligned}
$$


Нетривиальные фундаментальные скобки Пуассона даются формулами

$$
\begin{aligned}
\left\langle u(x, p), u^{*}(\xi, \eta)\right\rangle & =i \delta(x-\xi) \delta(p-\eta), \\
\left\langle\mathcal{A}_{s}(x), \mathcal{A}_{r}^{*}(y)\right\rangle & =i \delta_{r s} \delta(x-y), \\
\left\langle\widetilde{\mathcal{A}}_{s}(x), \widetilde{\mathcal{A}}_{r}^{*}(y)\right\rangle & =i \delta_{r s} \delta(x-y) .
\end{aligned}
$$

Остальные скобки равны нулю.

В качестве аналога уравнения (1) теперь следует взять уравнение

$$
\begin{gathered}
i \frac{\partial}{\partial t} \Psi(t)=\frac{1}{\varepsilon} \mathcal{H}\left(\sqrt{\varepsilon} \stackrel{2}{u}^{+}, \sqrt{\varepsilon} u^{-}, \sqrt{\varepsilon} \stackrel{2}{\mathcal{A}}^{+}, \sqrt{\varepsilon} \stackrel{1}{\mathcal{A}}^{-}, \sqrt{\varepsilon} \stackrel{2}{\mathcal{A}}^{+}, \sqrt{\varepsilon} \stackrel{1}{\mathcal{A}}^{-}\right) \Psi(t), \\
\Psi(t) \in \Gamma:=\Gamma_{s}\left(L^{2}\left(\mathbb{R}^{3} \times \mathbb{R}^{3}\right)\right)^{\otimes a_{0}} \otimes \Gamma_{s}\left(L^{2}\left(\mathbb{R}^{3} \times\{1,2\}\right)\right) \otimes \Gamma_{s}\left(L^{2}\left(\mathbb{R}^{3} \times\{1,2\}\right)\right),
\end{gathered}
$$

где $u_{a}^{ \pm}(x, p), \mathcal{A}^{ \pm}(x)$ и $\widetilde{\mathcal{A}}^{ \pm}(x)$ - операторы рождения-уничтожения в Г. Асимптотические решения этого уравнения можно построить с помощью метода комплексного ростка.

Благодарности. Авторы выражают благодарность Л. Д. Фаддееву, обратившему их внимание на важность формулы (41).

\section{Список литературы}

[1] V. P. Maslov, O. Yu. Shvedov. Russ. J. Math. Phys. 1994. V. 2. № 2. P. 217-234.

[2] Ф. А. Березин. Метод вторичного квантования. М.: Наука, 1986.

[3] В. П. Маслов. Комплексный метод ВКБ в нелинейных уравнениях. М.: Наука, 1977.

[4] В. А. Рубаков. Классические калибровочные поля. М.: Эдиториал УРСС, 1999.

[5] В. П. Маслов. Операторные методы. М.: Наука, 1973.

[6] М. В. Карасев, В. П. Маслов. Нелинейные скобки Пуассона. Геометрия и квантование. М.: Наука, 1991.

[7] V. P. Maslov, O. Yu. Shvedov. Russ. J. Math. Phys. 1995. V. 3. № 1. P. 65-80.

[8] L. D. Faddeev, A. J. Niemi. Magnetic geometry and confinement of electrically conducting plasmas. physics $/ 0003083$.

[9] В. П. Маслов, О. Ю. Шведов. ТМФ. 1995. Т. 104. № 2. С. 310-329.

[10] В. Гайтлер. Квантовая теория излучения. М.: ИЛ, 1956.

[11] Х. Хакен. Квантовополевая теория твердого тела. М.: Наука, 1980. 